\title{
Amortissement par viscosité de la houle sur un fond incliné dans un canal de largeur finie
}

\author{
Viscous damping of waves over an inclined bed \\ in a channel of finite width
}

\begin{abstract}
Dans un canal de largeur finie où se propage une houle de longuear donde grande par rapport it la profondeur, alcul de la dissipation d'énergie dans les conches voisines du fond, puis des parois. On en deduit une expression de l'amortissement de l'amplitude de la houle, qui confirme les résultats déjà connus pour le cas d'une largeur infinie. Cas où te fond du canal est légèrement incliné sur l'horizontale.
\end{abstract}

L'amortissement par viscosité des ondes progressives est important dans le cas des faibles profondeurs. Si les ondes se propagent dans un canal, la dissipation d'énergie se situe principalement dans les couches voisines de la paroi et du fond, à condition que la longueur d'onde soit grande par rapport à la profondeur. Si le canal est très large, on peut négliger l'effet de la paroi et, dans ce cas, Bresel a donné la solution correspondant à une profondeur constante [1] (1).

Afin d'obtenir la solution plus générale correspondant à un canal de largeur uniforme $b$, nous nous proposons de calculer le taux d'amortissement grâce à la méthode utilisée par URSELL [2] pour un canal profond. Nous supposerons que les variations de piofondeur sont toujours très lentes. La houle considérée est rigoureusement périodique dans le temps mais s'affaiblit au cours de sa progression.

Les axes de coordonnées $(x, y, z)$ sont choisis

\begin{abstract}
In a canal of finite and uniform width in which are propagated waves having a large wabelength in relation to the depth of water-the calculation of energy dissipation in the layers of water near the bed and near the walls, From this is deduced an expression of the damping of wave amplitude which confirm the results already obtained for the case in which the width is infinite. Instance in which the canal bed has a slight slope.
\end{abstract}

The attenuation of travelling waves due to viscosity is of importance in shallow water. In the case of waves propagated in a channel, energy is dissipated principally in the boundary layers on the walls and on the bed, provided that the wavelength is large compared to the depth. When the channel is very wide we may neglect the effect of the walls and the solution in this case, for a uniform depth, has been given by Breser. [1].

To obtain the more general result for a channel of uniform width $b$, we evaluate the rate of attenuation using the method employed by URSELL [2] for a deep channel. It will be assumed that the rate of change of depth is always small. The wave motion considered is strictly periodic in time but attenuated with distance.

We choose co-ordinates $(x, y, z)$ such that the

(1) Les chiffres [1] renvoient à la bibliographie publiée à la fin de cette note. 
de la façon suivante : $O x$ est dirigé suivant la longueur du canal, $\mathrm{O} y$ est vertical dirigé vers le bas, l'origine coïncidant avec l'intersection du niveau moyen et de l'une des parois du canal.

En fluide parfait, le potentiel d'une houle à deux dimensions à l'intérieur d'un petit intervalle $x_{1} \leqslant x \leqslant x_{2}$ est :

$$
\Phi=\frac{g A}{\sigma} \frac{\operatorname{ch} k(y-h)}{\operatorname{ch} k h} \cos (k x-\sigma t)
$$

avec :

$$
\begin{aligned}
& \sigma^{2}=g k \text { th } k h . \\
& k=2 \pi / \lambda . \\
& h=\text { la profondeur moyenne dans l'intervalle } \\
& \quad x_{1} \leqslant x \leqslant x_{2} . \\
& \mathrm{A}=\text { l'amplitude totale. }
\end{aligned}
$$

Supposons que dans un fluide de faible viscosité la vitesse $\vec{U}=(\mathrm{U}, \mathrm{V}, \mathrm{W})$ puisse être exprimée approximativement par :

$$
\overrightarrow{\mathrm{U}}=e^{-\mathrm{K} x} \operatorname{grad} \Phi
$$

Les équations du mouvement dans la théorie linéaire sont :

$$
\left(\frac{\partial}{\partial t}-\nu \nabla^{2}\right) \vec{U}=-\operatorname{grad}\left(\frac{p}{\rho}-g z\right)
$$

et :

$$
\operatorname{div} \overrightarrow{\mathrm{U}}=0
$$

en désignant par :

p: la pression,

$\varphi$ : la masse spécifique,

У: la viscosité cinématique.

Avec URSELL, considérons la fonction $\vec{u}(x, y, z)$ définie de la facon suivante :

$\vec{u}(x, y, z) e^{i \sigma t}=\overrightarrow{\mathrm{U}}(x, y, z, t)-\overrightarrow{\mathrm{iU}}\left(x, y, z, t+\frac{\pi}{2 \sigma}\right)$

$\mathrm{Si}$ :

$$
\vec{u}=\overrightarrow{u_{1}}+\overrightarrow{u_{2}}
$$

où $\overrightarrow{u_{1}}$ est irrotationnel :

$$
\overrightarrow{u_{1}}=\frac{i}{\sigma} \operatorname{grad}\left(\frac{p}{\rho}-g z\right)
$$

et :

$$
\left(i \sigma-y \overrightarrow{\left.\nabla^{2}\right)} u_{2}=0\right.
$$

axis $O x$ is along the length of the channel and Oy vertically downwards, with the origin at the intersection of the mean free surface and one wall of the channel.

From the inviscid theory the potential function for a two-dimensional wave over a small interval $x_{1} \leqslant x \leqslant x_{2}$ is given by :

$$
\Phi=\frac{g \mathrm{~A}}{\sigma} \frac{\operatorname{ch~} k(y-h)}{\operatorname{ch} k h} \cos (k x-\sigma t)
$$

where $\sigma^{2}=g k$ th $k h, k=2 \pi / \lambda, h$ is the mean depth in $x_{1} \leqslant x \leqslant x_{2}$ and $\mathrm{A}$ is the total wave amplitude. We suppose that in a fluid of small viscosity the velocity $\vec{U}=(U, V, W)$ is approximately :

$$
\overrightarrow{\mathrm{U}}=e^{-\mathrm{K} x} \operatorname{grad} \Phi
$$

The equations of motion in the linear theory are :

$$
\left(\frac{\partial}{\partial t}-v \nabla^{2}\right) \overrightarrow{\mathrm{U}}=-\operatorname{grad}\left(\frac{p}{\rho}-g z\right)
$$

and :

$$
\operatorname{div} \vec{U}=0
$$

where $p$ is the pressure, $p$ the density and $\nu$ the kinematic viscosity. Following Unseld, we consider the function $u(x, y, z)$ defined by :

$\vec{u}(x, y, z) e^{i \omega t}=\overrightarrow{\mathrm{U}}(x, y, z, t)-\overrightarrow{i \mathrm{U}}\left(x, y, z, t+\mathbf{2}_{\sigma}^{\pi}\right)$

and if :

$$
\vec{u}=\vec{u}_{1}+\vec{u}_{2}
$$

where $\overrightarrow{u_{1}}$ is irrotational, then :

$$
\overrightarrow{u_{1}}=\frac{i}{\sigma} \operatorname{grad}\left(\frac{p}{\rho^{\prime}}-g z\right)
$$

and :

$$
\left(i \sigma-v \nabla^{2}\right) \vec{u}_{2}=0
$$


Au fond du canal $\vec{u}=(u, v, w)=0$, et par conséquent :

$\vec{u}_{1}=\left(u_{0}, 0,0\right), \quad \overrightarrow{u_{2}}=\left(-u_{0}, 0,0\right)$ pour $y=h .(7)$

La solution de $u_{2 .}$ all voisinage des parois peut se déduire de l'équation :

$$
\left(i \sigma-\frac{\partial{ }^{2}}{\partial y^{2}}\right) u_{2}=0
$$

où $u_{2}=-u_{0}$ pour $y=h$, et où $u_{2}$ est petit pour $h-y>>\vee(y / \sigma)$.

On trouve facilement :

$$
\begin{aligned}
& u_{22}=-u_{0} e^{(1+i n(y-h)} \sqrt{(\sigma / 2) i n j} \\
& \left.\frac{\partial u_{0}}{\partial y}=-u_{0}(1+i) \sqrt{(\sigma / 2} v\right) e^{[(1+i)(y-h)} \sqrt{(\sigma / 2) /(j)]}
\end{aligned}
$$

Le taux de dissipation d'énergie mécanique par unité de temps dans un fluide incompressible est donnée par :

$$
\nu ? \iiint \mathrm{E} d x d y d z
$$

avec :

$$
\mathrm{E}=2\left(\frac{\partial \mathrm{U}}{\partial x}\right)^{2}+2\left(\frac{\partial \mathrm{V}}{\partial y}\right)^{2}+2\left(\frac{\partial \mathrm{W}}{\partial z}\right)^{2}+\left(\frac{\partial \mathrm{W}}{\partial y}+\frac{\partial \mathrm{V}}{\partial z}\right)^{2}+\left(\frac{\partial \mathbf{U}}{\partial z}+\frac{\partial \mathrm{W}}{\partial x}\right)^{2}+\left(\frac{\partial \mathrm{V}}{\partial x}+\frac{\partial \mathbf{U}}{\partial y}\right)^{2}
$$

On peut voir à ce sujet Lamb [3] et GoldsTEIN [4].

Le terme principal de l'expression (10) est :

\% $\iiint\left(\frac{\partial \mathbf{U}_{2}}{\partial y}\right)^{2} d x d y d z=\frac{1}{2} \times ? \iiint\left|\frac{\partial u \cdot}{\partial y}\right|_{(10 \text { bis })}^{2} d x d y d z$

et, d'après l'équation (9), la dissipation d'énergie dans la couche limite pendant le temps $2 \pi / \sigma$ est donnée par :

$$
\pi q \sqrt{y /(2 \sigma)} \iint\left|u_{0}\right|^{2} d x d z
$$

A l'intérieur de l'espace réduit $x_{1} \leqslant x \leqslant x_{2}$, nous pouvons écrire :

$$
\overrightarrow{u_{1}}=\operatorname{grad}\left[\frac{g \mathrm{~A}}{\sigma} \frac{\operatorname{ch} k(y-h)}{\operatorname{ch} k h} e^{-l i i x}\right]
$$

si bien que :

$$
\left.u_{0}\right\}=g \mathrm{~A} k /(\sigma \mathrm{ch} k h)
$$

On the bed of the channel, $\vec{u}=(u, v, b)=0$ and so :

$\vec{u}_{1}=\left(u_{0}, 0,0\right), \quad \vec{u}_{2}=\left(-u_{0}, 0,0\right) \quad$ on $y=h(7)$

The boundary layer solution for $u_{2,}$ is found from the equation :

$$
\left(i \sigma \cdots \cdots-\frac{\partial^{2}}{\partial y^{2}}\right) u_{2}=0
$$

where $u_{2}=-u_{0}$ on $y=h$ and $u_{2}$ is small for $h-\cdots\rangle>\sqrt{(y / \sigma)}$.

We readily find :

$$
u_{2}=-u_{0} e^{[(1+i)(y-h)} V^{(\sigma / 2, i)]}
$$

and :

$\frac{\partial u_{2}}{\partial y}=-u_{0}(1+i) \vee(\sigma / 2 \nu) e^{[(i+i)(y-h)} \sqrt{(\sigma / 2, p)]}$

The rate of dissipation of mechanical energy per unit time in an incompressible fluid is given by :

$$
\text { บ? } \iiint \mathrm{E} d x d y d z
$$

where:
The principal term in the expression $(10)$ is :

and from equation (9), the dissipation of energy in the boundary layer in time $2 \pi / \sigma$ is given by :

$$
\pi \bullet \sqrt{\nu /(2 \sigma)} \iint\left|u_{0}\right|^{2} d x d z
$$

Within the small region $x_{1} \leqslant x \leqslant x_{2}$ we may write :

$$
\overrightarrow{u_{1}}=\operatorname{grad}\left\lceil\frac{g \mathrm{~A}}{\sigma} \frac{\operatorname{ch} k(y-h)}{\operatorname{ch} k h} e^{- \text {tiia }}\right\rfloor
$$

so that :

$$
\left|u_{0}\right|=g \mathrm{~A} k /(\sigma \mathrm{ch} k h)
$$

$$
\vee \varphi \iiint\left(\frac{\partial \mathrm{U}_{2}}{\partial y}\right)^{2} d x d y d z=\frac{1}{2} \vee \varphi \iiint\left|\frac{\partial u_{2}}{\partial y}\right|^{2} d x d y d z
$$


et, en calculant l'intégrale de l'expression (11), la dissipation d'énergie, pendant l'intervalle de lemps $2 \pi / \sigma$, dans la zone $x_{1} \leqslant x \leqslant x_{2}, 0 \leqslant z \leqslant b$ du plan $y=h$ est :

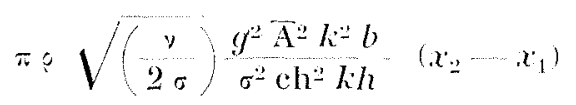

en appelant $\bar{A}$ l'amplitude moyenne de la houle dans cette zone.

Suivant la mène méthode, nous pouvons calculer le taux de perte d'énergie dans les couches voisines des parois du canal. Sur le plan $z:=0$,

$$
\vec{u}=(u, v, w)=0
$$

et :

$$
\overrightarrow{u_{1}}=\left(u_{0}, b_{0}, w_{0}\right) \quad \overrightarrow{u_{2}}=-\left(u_{i}, v_{0}, w_{0}\right)
$$

D’après l'écruation :

$$
\left(i \sigma-\frac{\partial z^{2}}{\partial z^{2}}\right) u_{2,2}=0
$$

on trouve :

$$
u_{22}=--u_{0} e-(1+i) \sim \sqrt{(0 / 2 t)}
$$

el :

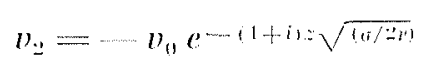

Ainsi :

$$
\partial u_{z}=u_{0}(1+i) \sqrt{(\sigma / 2 y)} e^{-(1+i) \cdot \sqrt{\left(1+/ n^{\prime}\right)}}
$$

(c) :

$$
\frac{\partial n_{2}}{\partial z}=v_{0}(1+i) \vee(\pi / 2, y) e-(1+i)=\sqrt{ }(\sigma / 2)(16)
$$

Le terme principal de lintégrale (10) dû̀ à la couche limite à la paroi $z=0$ est : and evaluating the integral in (11), the dissipation in time $2 \pi / \sigma$ in the region $x_{1} \leqslant x \leqslant x_{2}$, $0 \leqslant z \leqslant b$ of the plane $y=h$ is :

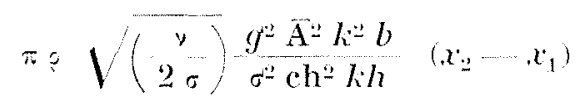

where $\mathrm{A}$ is the mean wave amplitude in the region.

In the same way we find the rate of loss of energy in the boundary layers on the walls of the channel. On $z=0$,

$$
\vec{u}=(u, v, u)=0
$$

and so :

$$
\overrightarrow{u_{1}}=\left(u_{0}, v_{0}, w_{0}\right) \quad \overrightarrow{u_{2}}=\ldots\left(u_{0}, v_{0}, w_{0}\right)
$$

From the equation :

$$
\left(i \sigma \cdots, \frac{\partial z}{\partial z^{2}}\right) u_{i=0}=0
$$

we find :

$$
u_{2}=-u_{0} e-1+i n \sqrt{(i) / 2 m)}
$$

and similarly :

$$
v_{2.2}=-v_{0} e-(1+i) z V^{(a / 2, i)}
$$

Thus :

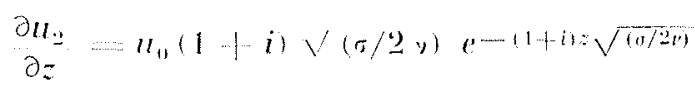

and :

$$
\frac{\partial v_{2}}{\partial z}=l_{0}(1+i) \vee(\sigma / 2 y) e-(1+i) z \sqrt{(\sigma o t 2 i i)}
$$

The principal contribution to the integral (10) in the boundary layer on the wall $z=0$ is :

$$
\vee \rho \iiint\left[\left(\frac{\partial U_{2}}{\partial z}\right)^{2}+\left(\frac{\partial V_{2}}{\partial z}\right)^{2}\right] d x d y d z=-\frac{1}{2} \times q \iiint\left[\left|\frac{\partial u_{2}}{\partial z}\right|^{2}+\left|\begin{array}{c}
\partial v_{2} \\
\partial z
\end{array}\right| d x d y d z\right.
$$

et compte tenu des expressions (15) et (16), la dissipation pendant le temps $2 \pi / \sigma$ est :

$$
\pi \circ \sqrt{(y / 2 \sigma)} \iint\left(\left|u_{0}\right|^{2}+\left|v_{0}\right|^{2}\right) d x d y
$$

De l'équation (12), on tire :

$$
\left|u_{0}\right|^{22}+\left|v_{0}\right|^{2}=\frac{g^{2} A^{2} k^{2}}{\sigma^{2}} \frac{\operatorname{ch} 2 k(y-h)}{\operatorname{ch}^{2} k h}
$$

and substituting the expressions (15) and (16), the dissipation in time $2 \pi / \sigma$ is :

$$
\dot{\pi} \sigma \sqrt{(\nu / 2 \sigma)} \iint\left(\left|u_{0}\right|^{2}+\left|v_{0}\right|^{2}\right) d x d y
$$

From equation (12) we have :

$$
\left|u_{0}\right|^{2}+\left|b_{0}\right|^{2}=\frac{g^{2} \mathrm{~A}^{2} l_{i}^{2}}{\sigma^{2}} \frac{\operatorname{ch} 2 l^{2}(y-h)}{\operatorname{ch}^{2} k h}
$$


d'où l'on déduit que la dissipation totale d'énergie pendant l'intervalle de temps $2 \pi / \sigma$ dans la zone $x_{1} \leqslant x \leqslant x_{2}, \quad 0 \leqslant y \leqslant h$ du plan $z=0$ est égale à :

$$
\pi \cdot \sqrt{\left(\frac{y}{2 \sigma}\right)} \frac{g^{2} \overline{\mathrm{A}}^{2} k}{\sigma^{2}} \text { th } \operatorname{kh}\left(x_{2}-x_{1}\right)
$$

Au cours d'une période de la houle $(2 \pi / \sigma)$, l'énergie traversant le plan $x=$ constante est :

$$
-? \int_{0}^{2 \pi / \sigma} \int_{0}^{b} \int_{0}^{h} \frac{\partial \Phi}{\partial t} \frac{\partial \Phi}{\partial x} d y d z d t
$$

Si l'on tient compte de l'équation (1), cette expression devient :

$$
\frac{\pi \circ b g \mathrm{~A}^{2}}{2 k}\left(1+\frac{2 k h}{\operatorname{sh} 2 k h}\right)
$$

Il est évident que la différence entre les énergies traversant les plans $x=x_{1}=x_{2}$, est égale à l'énergie dissipée dans la zone $x_{1} \leqslant x \leqslant x_{2}$; par conséquent, les équations (13), (19) et (20) permettent d'écrire :

$$
\begin{aligned}
& \frac{\pi p b g}{2 k}\left(1+\frac{2 k h}{\operatorname{sh} 2 k h}\right)\left(\mathrm{A}_{1}^{2}-\mathrm{A}_{2}{ }^{2}\right) \\
& =\pi \cdot \sqrt{(2 \sigma)\left(x_{2}-x_{1}\right)} \frac{g \cdot \mathrm{A}^{2} k}{\sigma^{2}}\left(2 \text { th } k h+\frac{k b}{\mathrm{ch}^{2} k h}\right)
\end{aligned}
$$

ou :

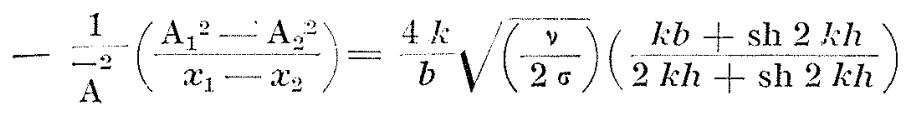

D'après l'équation (2) :

$$
\mathrm{K}=-\frac{1}{\mathrm{~A}} \frac{d \mathrm{~A}}{d x}
$$

et si nous faisons tendre $x_{2}$ vers $x_{1}$ dans l'équation (21) nous obtenons :

$$
\mathrm{K}=\frac{2 k}{b} \sqrt{\left(\frac{v}{2 \sigma}\right)\left(\frac{k b+\operatorname{sh} 2 k h}{2 k h+\operatorname{sh} 2 k h}\right)}
$$

Ceci concorde avec le résultat cité dans les Proceedings of the Conference on Coastal Engineering 1950 , page 206 . Lorsque la largeur $b$ du canal devient grande, $K$ tend vers le terme principal de la solution donnée par M. Biesel. Pour de petites valeurs de $k h, \mathrm{~K}$ ne diffère que par un facteur $\pi / 4$ du résultat obtenu par KevLEGAN [5] pour l'amortissement de l'onde solitaire.

Supposons maintenant que le fond du canal soit légèrement incliné sur l'horizontale. En négligeant provisoirement l'effet de la viscosité, le and so the total dissipation in time $2 \pi / \sigma$ in the region $x_{1} \leqslant x \leqslant x_{2}, 0 \leqslant y \leqslant h$ of the plane $z=0$ is found to be :

$$
\pi \bullet \sqrt{\left(\frac{y}{2 \sigma}\right)} \frac{g^{2} \bar{A}^{2} k}{\sigma^{2}} \text { th } \operatorname{kh}\left(x_{2}-x_{1}\right)
$$

The energy transmitted across the plane $x=$ constant in one wave period $2 \pi / \sigma$ is :

$$
-\rho \int_{0}^{2 \pi / \sigma} \int_{0}^{b} \int_{0}^{h} \frac{\partial \Phi}{\partial t} \frac{\partial \Phi}{\partial x} d y d z d t
$$

Substituting from equation (1), this becomes :

$$
\frac{\pi ? b g A^{2}}{2 k}\left(1+\frac{2 k h}{\operatorname{sh} 2 k h}\right)
$$

It is clear that the difference between the energy crossing the planes $x=x_{1}$ and $x=x_{2}$ is equal to the energy dissipated in the region $x_{1} \leqslant x \leqslant x_{2}$, and so from equations (13), (19) and (20) we have :

$$
\begin{aligned}
& \frac{\pi \circ b g}{2 k}\left(1+\frac{2 k h}{\operatorname{sh} 2 k h}\right)\left(A_{1}{ }^{2}-A_{2}{ }^{2}\right) \\
& =\pi \circ \sqrt{\left(\frac{\nu}{2 \sigma}\right)\left(x_{2}-x_{1}\right) \frac{g_{2} \bar{A}^{2} k}{\sigma^{2}}\left(2 \operatorname{th} k h+\frac{k b}{c^{2} k h}\right)}
\end{aligned}
$$

or :

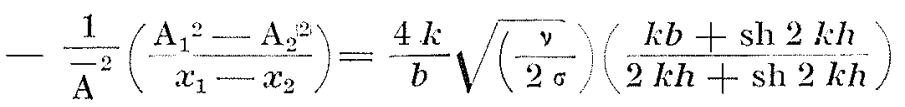

From equation (2) :

$$
\mathrm{K}=-\frac{1}{\mathrm{~A}} \frac{\mathrm{dA}}{d x}
$$

and thus letting $x_{2} \rightarrow x_{1}$ in equation (21) we have :

$$
\mathrm{K}=\frac{2 k}{b} \sqrt{\left(\frac{v}{2 \sigma}\right)}\left(\frac{k b+\operatorname{sh} 2 k h}{2 k h+\operatorname{sh} 2 k h}\right)
$$

This agrees with the result quoted in the Proceedings of the Conference on Coastal Engineering 1950, p. 206 . When the width of the channel $b$ becomes very large, $\mathrm{K}$ tends to the principal term in the solution given by BIESEL. For small values of $k h, \mathrm{~K}$ differs only in a factor $\pi / 4$ from the result obtained by KEULEGaN [5] for the damping of the selitary wave.

Suppose now that the channel bed is inclined at a small angle to the horizontal. Neglecting for the moment the effect of viscosity, the rate 
flux d'énergie à travers un plan quelconque $x=$ constante doit être toujours le même. Done :

$$
A^{2} c\left(1+\frac{2 k h}{\operatorname{sh} 2 k h}\right)=A_{0}{ }^{2} c_{0}\left(1+\frac{2 k_{0} h_{0}}{\operatorname{sh} 2 k_{0} h_{0}}\right)
$$

en désignant par c la célérité de la houle; et puisque on peut écrire :

$$
\begin{gathered}
\frac{\lambda}{\lambda_{0}}=\frac{c}{c_{0}}=\text { th } k h / \text { th } k_{0} h_{0} \\
A=A_{0} \sqrt{\left(\frac{\operatorname{th~} k_{0} h_{0}+k_{0} h_{0} \operatorname{sech}^{2} k_{0} h_{0}}{\operatorname{th} k h+k h \operatorname{sech}^{2} k h}\right)}
\end{gathered}
$$

Par conséquent, la modification de l'amplitude de la houle le long d'un intervalle $d x$ est donné, en négligeant la viscosité, par :

$$
d \mathrm{~A}=\mathrm{A}\left(\operatorname{th} k h+k h \operatorname{sech}^{2} k h\right)^{1 / 2} \frac{d}{d x}\left(\text { th } k h+k h \operatorname{sech}^{2} k h\right)-1 / 2 d x
$$

De l'équation (22), on peut déduire l'effet de la viscosité, soit :

$$
d \mathrm{~A}=-\mathrm{A} \frac{2 k}{b} \sqrt{\left(\frac{v}{2 \sigma}\right)\left(\frac{k b+\operatorname{sh} 2 k h}{2 k h+\operatorname{sh} 2 k h}\right)} d x
$$

Si l'on fait la somme des deux différentielles (23) et (24) et si l'on intègre, l'amplitude de la houle en un point quelconque est : of flow of energy must be the same past any plane $x=$ constant. Thus :

$$
\mathrm{A}^{3} c\left(1+\frac{2 k h}{\operatorname{sh} 2 k h}\right)=\mathrm{A}_{0}{ }^{2} c_{0}\left(1+\frac{2 k_{0} h_{0}}{\operatorname{sh} 2 k_{0} h_{0}}\right)
$$

where $c$ is the wave velocity, and since:

$$
\frac{\lambda}{\lambda_{0}}=\frac{c}{c_{0}}=\text { th } k h / \text { th } k_{0} h_{0}
$$

then :

$$
\mathrm{A}=-\mathrm{A}_{0} \sqrt{\left(\frac{\mathrm{th} k_{0} h_{0}+k_{0} h_{0} \operatorname{sech}^{2} k_{0} h_{0}}{\text { th } k h+k h \operatorname{sech}^{2} l h}\right)}
$$

Thus the change in wave amplitude in an interval $d x$, neglecting viscosity, is given by :

From equation (22) the effect of viscosity is given by :

$$
d \mathrm{~A}=-\mathrm{A} \frac{2 k}{b} \sqrt{\left(\frac{y}{2 \sigma}\right)}\left(\frac{k b+\operatorname{sh} 2 k h}{2 k h+\operatorname{sh} 2 k h}\right) d x
$$

Adding the differentials in (23) and (24) and integrating, the wave amplitude at any point is :

$$
\mathrm{A}=\mathrm{A}_{0} \sqrt{\left(\frac{\operatorname{th} k_{0} h_{0}+k_{0} h_{0} \operatorname{sech}^{2} k_{0} h_{0}}{\operatorname{th} k h+k h \operatorname{sech}^{2} k h}\right)} e^{\int_{h_{0}}^{h}-(2 k / b) \sqrt{v / 2 \sigma}\left(\frac{k b+\operatorname{sh} 2 h h}{2 k h+\operatorname{sh} 2 k h}\right) d x}
$$

Si l'on pose :

$$
h=a-s x
$$

en désignant par a la profondeur à l'origine et par $s$ la pente du fond, l'intégrale peut se calculer en se servant de la relation :

$$
k_{0} \text { th } k_{0} h_{0}=k \text { the } k \text { h }=\sigma^{2} / g,
$$

où $k=\frac{2 \pi}{\lambda}$. L'équation (25) devient :
If we write :

$$
h=a-s x
$$

where $a$ is the depth at the origin and $s$ is the slope of the bed, the inlegral can be evaluated using the relation :

$$
k_{0} \text { th } k_{0} h_{0}=k \text { th } k h=\sigma^{2} / g,
$$

where $k=2 \pi / \lambda$. Equation (25) becomes :

$$
A=A_{0}\left(\frac{\operatorname{th~} k_{0} h_{0}+k_{0} h_{0} \operatorname{sech}^{2} k_{0} h_{0}}{\operatorname{th} k h+k h \operatorname{sech}^{2} k h}\right)^{1 / 2}\left(\frac{k^{2}\left(g^{2} k_{0}{ }^{2}-\sigma^{1}\right)}{k_{0}{ }^{2}\left(g^{2} k^{2}-\sigma^{4}\right)}\right)^{(1 / s b) \sqrt{(v / 2 \sigma)}} e^{-(1 / s)\left(k-k_{0}\right) \sqrt{v^{2} / 2 \sigma}}
$$

la valeur de $k$ en chaque point pouvant être déduite des équations (26) el (27).

Nous avons admis que la célérité de la houle était en chaque point égale à la célérité d'une houle de mêne période dans un eanal ayant uniformément la profondeur correspondant à celle where $k$ at any point may be found from equations (26) and (27).

We have assumed that the wave velocity at any point is equal to the velocity of a wave of the same period in a channel of the same uni- 
du point considéré. Le résultat n’est done valable que pour les petites valeurs de la pente $s$.

Nous nous devons d'exprimer notre reconnaissance au Directeur de la Recherche Hydraulique qui a bien voulu nous autoriser à publier ce travail. form depth. The result is therefore valid only for small values of the slopes $s$.

I am indebted to the Director of Hydraulies Research for permission to publish this work.

\section{J. N. Hun't,}

Hydraties Research station-DSIR Howbery Park, Wallingford, Berlss

\section{BIBLIOGRAPHIE}

1. F. Buser, 1949, la Houtle Blanche, vol. 4, $11^{\prime 5} 5$, p. 630 .

2. F. Unser., 1952, Proc. Roy. Soc. A 214 , p. 79.

3. Lams, 1982, Hydrodymamies, art. 329.

4. Goupstax, 1938, Modern Developments in Fluid Dynamics, alt. 37.

5. Kevegax, 1948, Journal of Researeh of the National Bureau of Standards, 40, p. 487.

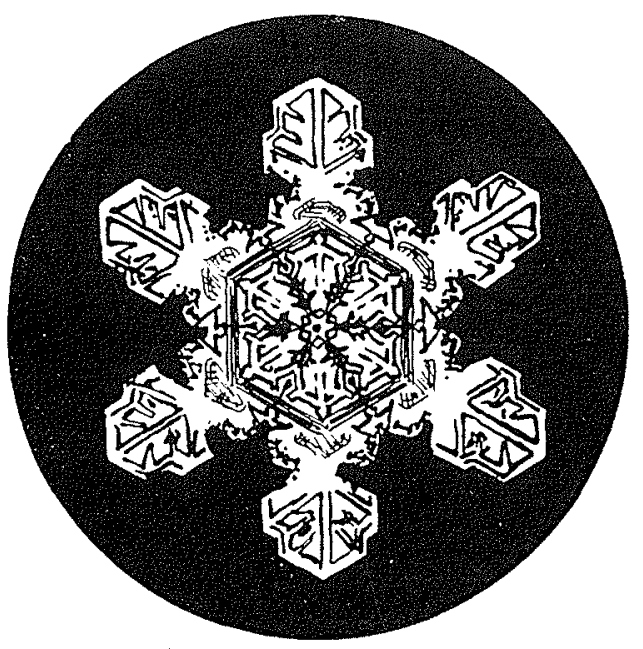

